\title{
Editorial
}

\section{Challenges for the Future Mobile Communication Systems}

\author{
Yujin Lim, ${ }^{1}$ Hideyuki Takahashi, ${ }^{2}$ Fan $W u,{ }^{3}$ and Rossana M. de Castro Andrade ${ }^{4}$ \\ ${ }^{1}$ Department of Information Technology Engineering, Sookmyung Women's University, Seoul 04310, Republic of Korea \\ ${ }^{2}$ Research Institute of Electrical Communication, Tohoku University, Sendai 980-8577, Japan \\ ${ }^{3}$ Department of Computer Science and Engineering, Shanghai Jiao Tong University, Shanghai 200240, China \\ ${ }^{4}$ Department of Computer Science, Federal University of Ceará, 2853 Benfica, Fortaleza, CE, Brazil
}

Correspondence should be addressed to Yujin Lim; yujin91@sookmyung.ac.kr

Received 18 April 2017; Accepted 18 April 2017; Published 30 May 2017

Copyright (C) 2017 Yujin Lim et al. This is an open access article distributed under the Creative Commons Attribution License, which permits unrestricted use, distribution, and reproduction in any medium, provided the original work is properly cited.

As the wireless data traffic has been increased explosively, the future mobile communication system that can accommodate the traffic demand in a cost-effective and optimal manner attracts more and more attention in both academia and industry. Many standards bodies, countries, and major companies have proposed visions for the future mobile communication system and there have been a lot of proposals from the architectural changes to specific algorithms. For example, ITU specified the key performance requirements of $5 \mathrm{G}$ networks and 3GPP presented the roadmap for $5 \mathrm{G}$ standard deployment. NGNM presented use cases of $5 \mathrm{G}$ applications.

Future mobile communication systems will need to interconnect heterogeneous mobile communication systems and technologies and get them to work together so as to optimize services requirements and operational conditions. For example, software defined network is supposed to be deployed for core and access networks, machine-type communications will prevail, and self-organized mechanisms are introduced to increase network performance and reduce operational costs under highly variable and unpredictable network conditions. Even though a significant number of studies taking evolutionary approaches and revolutionary approaches are in progress to give shape to the vision of the future mobile communication system, more efforts are still required to crystallize the future mobile communication system.

The scope of this special issue is in line with recent contributions from academia and industry on the recent activities that tackle the technical challenges to concretize the future mobile communication system. For the current issue, we are pleased to introduce a collection of papers covering a range of topics as follows:

(i) Technologies for connected vehicle systems

(ii) Technologies for cellular networks

(iii) Technologies for wireless multihop networks

(iv) Technologies for underwater wireless networks

(v) Technologies for future mobile communication systems

\section{Acknowledgments}

As always, we appreciate the high quality submissions from authors and the support of the community of reviewers.

Yujin Lim

Hideyuki Takahashi

Fan Wu

Rossana M. de Castro Andrade 

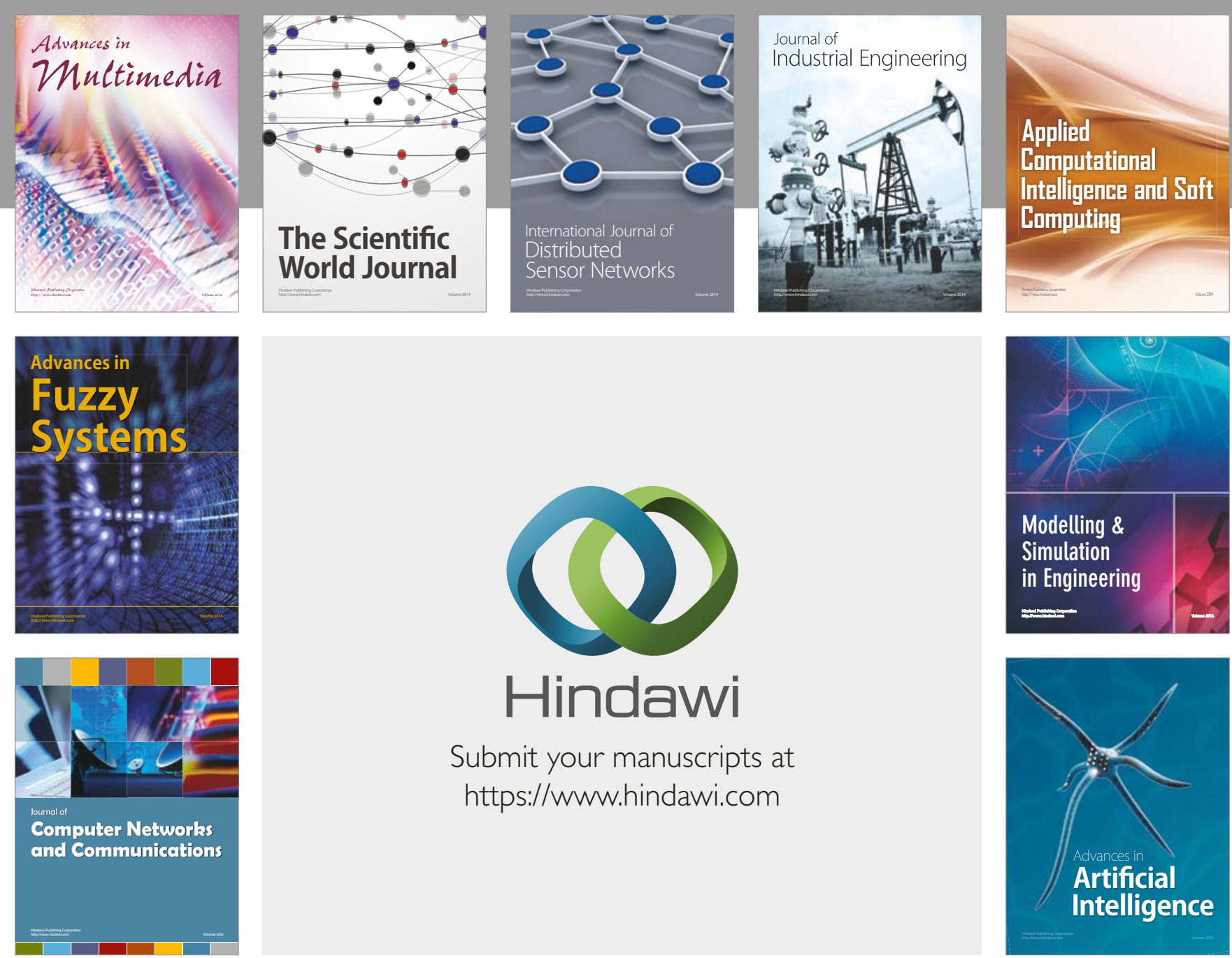

\section{Hindawi}

Submit your manuscripts at

https://www.hindawi.com
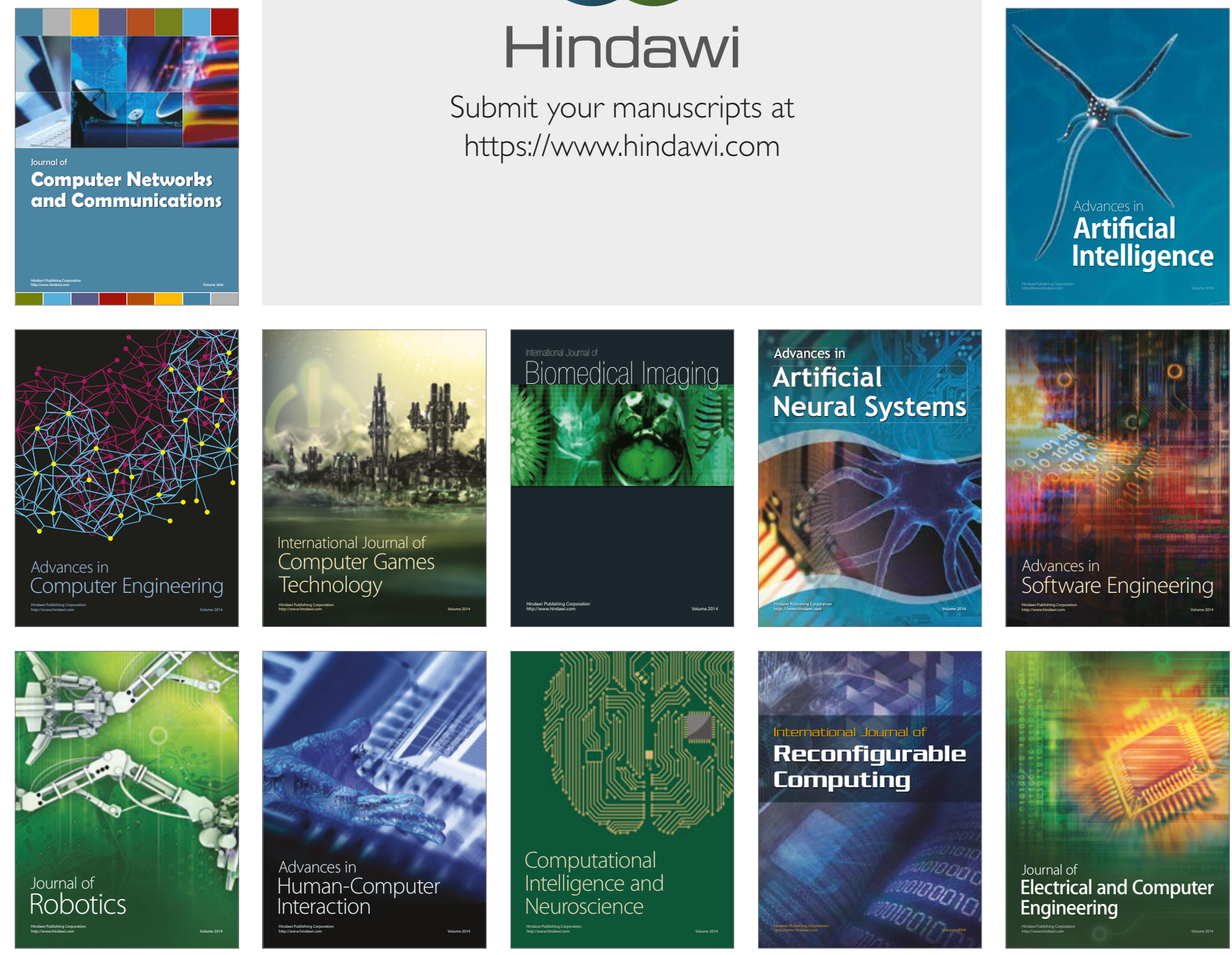